
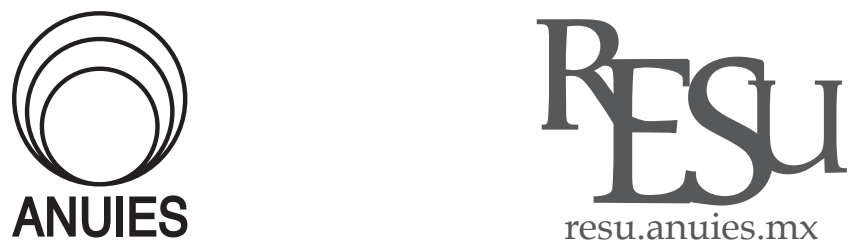

RESEÑA

\title{
La universidad pública y la importancia de las incubadoras como mecanismos para impulsar el desarrollo regional*
}

Public universities and the importance of incubators as mechanisms to boost regional development

\section{Wendy Ovando Aldana**}

* Canales García, Azalea (2018). Redes de conocimiento y desarrollo regional en el marco de la incubación de empresas universitarias. El caso de la UAEMex. México: ANUIES.

** Facultad de Economía de la Universidad Autónoma del Estado de México.

Correo electrónico: gwenovando@live.com.mx

$\square^{1}$ objetivo del libro, obra de Azalea Canales García y que a continuación $\checkmark$ reseño, radica en analizar la estructura de las posibles redes de conocimiento generadas por la interacción de las incubadoras de la Universidad Autónoma del Estado de México (uAemex), así como los mecanismos de transferencia de ese conocimiento para impulsar el desarrollo regional del Estado de México. Es necesario indicar que se trata de un texto de fácil lectura para comprender el análisis de este tema: la generación y la transmisión de conocimiento a partir de las conexiones reticulares, la importancia económica de las incubadoras para cualquier nación (en específico para México), el nuevo papel de las universidades públicas como transmisoras de conocimiento a partir de la asesoría de proyectos de inversión, o bien, la conexión entre las redes de conocimiento - que se producen entre las incubadoras - y el territorio como mecanismo de impulso del desarrollo regional.

Antes de comenzar con la descripción de la estructura del libro, me parece importante señalar que el título describe la trascendencia del conoci- 
miento como un factor de producción que origina innovación; asimismo advierte cómo, a partir de conexiones heterogéneas, es posible transmitirlo entre las organizaciones y la sociedad. Es decir, se puede aprender de forma colectiva mediante las redes de conocimiento: ¿cómo es que las universidades juegan un papel preponderante en estas redes? El libro menciona el enfoque teórico que permite incorporar a la universidad en el análisis de tales redes: el modelo triple hélice. Esta perspectiva indica que existe una interacción entre tres agentes principales que crean y transfieren conocimiento: industrias, gobierno y universidad; los tres elementos se conectan reticularmente y forman estructuras como las incubadoras cuya relevancia, de acuerdo con el documento, versa sobre la transmisión de conocimiento en tanto motor para crear empleos y negocios que pueden no ser innovadores, pero cuando lo son influyen en el desarrollo económico que, en el título, se denomina desarrollo regional. Como se puede detectar, el título enuncia los componentes más reveladores en torno al tema: redes de conocimiento, universidad, incubadoras y desarrollo regional.

La autora seleccionó a las incubadoras de la UAEMex porque en el momento en que hizo el estudio, hacia 2011, dicha universidad contaba con 12 unidades de asesoramiento, las cuales representaban, de manera conjunta, la red de incubadoras más grande en México. Asimismo, en la introducción, la obra plantea tres hipótesis, de las cuales sobresale la última porque encierra el objetivo de la investigación: la red de incubadoras de la UAEMex tiene una aportación marginal en la generación y la difusión de conocimiento para el desarrollo regional (mexiquense).

El documento se encuentra organizado en siete capítulos: los tres primeros representan el andamiaje teórico que sustenta la investigación, el cuarto describe la metodología empleada para evaluar las hipótesis, y los últimos tres apartados versan sobre la investigación aplicada.

El capítulo primero - redes, conocimiento y desarrollo regional- estudia el papel preponderante que tiene el conocimiento como factor de la producción y como elemento capaz de generar el crecimiento económico a partir de la creación de productos con alto valor agregado. El conocimiento ya no es una variable exógena dentro de las funciones de la producción; en esta nueva etapa, denominada Economía Basada en el Conocimiento, el factor cognitivo termina siendo un componente endógeno, resultado de la relación entre diferentes agentes. De igual forma, se explican los fundamentos de la Teoría de Redes Sociales, el significado de una red como pieza fundamental de estudio, los elementos y tipos redes, y el análisis de redes sociales como estructuras consecuencia de la interacción entre diversos actores (sociedad, organizaciones, etc.). Al mismo tiempo, la lec- 
tura explica las características que constituyen una red de conocimiento - cuyo principal objetivo es la innovación y el crecimiento económico-, entre otras, las dimensiones, los mecanismos de integración y el desempeño de la red. El primer capítulo cierra con la perspectiva del desarrollo regional endógeno haciendo énfasis en el valor que tienen los recursos de un territorio determinado (región) para fortalecer su economía.

Enseguida, en el capítulo segundo -Modelo Triple Hélice, capital relacional y universidad emprendedora - , la autora describe el modelo Triple Hélice y el resultado de la interacción entre los agentes que participan en este vínculo tripartita (universidad, industria y gobierno): favorecer e impulsar actividades innovadoras y crecimiento económico; posteriormente refiere los tres niveles de conexión que pueden generarse entre los actores principales del modelo Triple Hélice, para indicar que el idóneo es el equilibrado porque - al no existir un agente predominante en la interacción uno de los resultados de la conexión balanceada entre universidad, sector productivo y gobierno lo representan las incubadoras. Pero no sólo eso, la relación entre estos tres elementos del enfoque de Triple Hélice suscita un capital relacional, que deriva en innovación y crecimiento económico. El elemento objeto de análisis de esta investigación radica en la universidad, por lo tanto, para explicar su significancia se recurre a una vertiente denominada Universidad Emprendedora que resalta no sólo las funciones básicas y conocidas que ésta desempeña (docencia, investigación, solución a problemas sociales), sino además la disposición para la sociedad de incubadoras que impulsen la innovación, el emprendimiento y el apoyo a los empresarios de la región.

El capítulo tercero (incubadoras de empresas) se centra en la definición de las incubadoras, pero además describe sus tipos y la historia de su surgimiento. Al mismo tiempo, el texto señala los diversos modelos de incubación que van desde la primera hasta la tercera generación; las incubadoras asociadas al Modelo Lógico y la Teoría del Cambio representan las más avanzadas por el empleo de ambientes virtuales y la conformación de estructuras en forma de redes. Dos incubadoras internacionales son retomadas como ejemplos en el documento: el Valle del Silicio en E.U., prototipo de una economía desarrollada, y las que se hallan en Brasil para el contexto latinoamericano. Esta sección finaliza con la situación de las incubadoras de empresas en México; relata la secuencia cronológica del surgimiento y el estado actual de las incubadoras mexicanas destacando tres etapas de desarrollo de la incubación: una denominada como periodo incipiente de impulso al emprendimiento (años noventa), y otra de desarrollo de programas gubernamentales y crecimiento de incubadoras (pri- 
mer decenio de 2000), y una más acentuada por la implantación de estrategias (2013 a la fecha). Aun y cuando se sabe de la trascendencia de las incubadoras, no existe suficiente investigación al respecto, por lo que esta obra suma información al análisis del tema en México.

En el capítulo cuarto, la Dra. Canales García se enfoca en la descripción metodológica para hallar las redes sociales de conocimiento, específicamente en las incubadoras de la UAEMex y su ulterior vínculo con el territorio mexiquense en el impulso del desarrollo regional. Resulta interesante observar que sólo 10 de las 12 incubadoras, que en su momento operaban en la universidad de estudio, entregaron información de su interconexión a partir de un cuestionario semiestructurado diseñado por la autora y dirigido a los coordinadores de incubadoras. Vale la pena revisar la metodología dado que la Ciencia Económica exige la formalización matemática de sus estudios y ésta es una propuesta de carácter multidisciplinario que analiza un tema de interés económico, sociológico, político, administrativo, la cual en términos generales no requiere del rigor matemático, sino que basta con el análisis de redes mediante su representación gráfica. Es decir, existen técnicas de estudio alternativas para estudiar diversas variables, en este caso las redes de conocimiento y su relación con el desarrollo regional.

El capítulo quinto muestra parte de los resultados, retoma la parte teórica expuesta en los capítulos anteriores y explica, mediante una representación gráfica y estadística, que las incubadoras de la universidad mexiquense presentan conexiones poco enlazadas, semejantes a uno de los tipos de redes descritos en el capítulo primero: en estrella. En otras palabras, existe un actor central que, si no funciona correctamente, causa problemas en toda la red (en este caso, la incubadora central es la que se ubica en la ciudad de Toluca), lo cual podría frenar la generación y la transmisión del conocimiento porque los enlaces se concentran en una sola incubadora.

En el capítulo sexto se analizan las dimensiones de las redes sociales: institucional, morfológica, estructural y dinámica. Lo anterior resulta relevante porque se establecen las causas del bajo nivel de fortalecimiento de la red entre universidad, empresas y gobierno, entre otras, los trámites burocráticos, el tiempo de respuesta para apoyar a los emprendedores, el desconocimiento por parte de la sociedad acerca de la utilidad que reportan las incubadoras, etc. Al mismo tiempo, se identifica entre las incubadoras de la UAEMex el nulo intercambio de experiencia, de recursos humanos y de infraestructura. No obstante, la comunicación entre las incubadoras, a través de reuniones constantes, genera lazos de confianza entre los coordinadores. 
Finalmente, en el capítulo séptimo se encuentra el análisis de redes sociales sobre el desempeño organizacional, funcional y económico. Las incubadoras de empresas de la UAEMex están vinculadas con la geografía económica y aparecen cercanas a actividades económicas esenciales, sin embargo, los negocios a los que se orientan tienen una aportación marginal en el desarrollo de la región, pues asesoran negocios tradicionales con escaso o nulo valor agregado.

En síntesis, la investigación que presenta la Dra. Azalea Canales García resulta una propuesta diferente para analizar las redes de conocimiento, en específico, las redes sociales entre un grupo de incubadoras universitarias y su conexión con el desarrollo regional, es decir, realiza una combinación de técnicas de análisis: cuantitativa, gráfica y cualitativa. A pesar de abandonar las exigencias de la ortodoxia económica en cuanto al empleo de modelos y su formalización matemática, no se aleja de la rigurosidad teórica. La autora revisa a profundidad los fundamentos que dan soporte a los hallazgos y conclusiones; retoma, principalmente, el modelo de Triple Hélice, pero combina esta perspectiva con el desarrollo regional endógeno para justificar la importancia de la interacción de tres actores (universidad, empresarios, gobierno) en el impulso del crecimiento y el desarrollo económicos. Al analizar las incubadoras, la obra hace recordar las teorías económicas como la schumpeteriana, en la que innovación y desarrollo económico van de la mano.

No está de más señalar que los resultados de este libro no pueden ser generalizados a otros contextos, ya que las redes sociales entre diferentes actores en un espacio disímil presentarán características propias de las interacciones sociales gestadas. Es más, la propia teoría del desarrollo endógeno recuerda que los recursos de cada territorio son disímiles al comparar un espacio con otro. Aunado a lo anterior, se debe recordar que se estudian las incubadoras de una universidad pública y quizá en el ámbito privado los resultados pueden variar. Al mismo tiempo, esta investigación es de corte transversal, o bien de un análisis estático, pero permite tener un escenario de las incubadoras de la UAEMex y su relación con el desarrollo regional. Para quienes son responsables de las tomas de decisiones, o bien, los encargados de las políticas públicas, este libro marca un antecedente sobre lo que no está marchando correctamente en cuestión de innovación y de desarrollo regional. 
